\title{
Direct Imaging Of Long Period Radial Velocity Targets With NICI
}

\author{
Graeme S. Salter ${ }^{1}$, Chris G. Tinney ${ }^{1}$, Robert A. Wittenmyer ${ }^{1}$, \\ James S. Jenkins ${ }^{2}$, Hugh R.A. Jones ${ }^{3}$, and Simon J. O'Toole ${ }^{4}$ \\ ${ }^{1}$ Department of Astrophysics, University of New South Wales, \\ Kensington Campus, 2034, NSW, Australia \\ email: g.salter@unsw.edu . au \\ ${ }^{2}$ Universidad de Chile, Camino el Observatorio 1515, \\ Las Condes, Santiago, Chile, Casilla 36-D \\ ${ }^{3}$ University of Hertfordshire, Centre for Astrophysics Research, Science and Technology \\ Research Institute, College Lane, AL10 9AB, Hatfield, UK \\ ${ }^{4}$ Australian Astronomical Observatory, PO Box 915, North Ryde \\ 1670, NSW, Australia
}

\begin{abstract}
We are finally entering an era where radial velocity and direct imaging parameter spaces are starting to overlap. Radial velocity measurements provide us with a minimum mass for an orbiting companion (the mass as a function of the inclination of the system). By following up these long period radial velocity detections with direct imaging we can determine whether a trend seen is due to an orbiting planet at low inclination or an orbiting brown dwarf at high inclination. In the event of a non-detection we are still able to put a limit on the maximum mass of the orbiting body. The Anglo-Australian Planet Search is one of the longest baseline radial velocity planet searches in existence, amongst its targets are many that show long period trends in the data. Here we present our direct imaging survey of these objects with our results to date. ADI Observations have been made using NICI (Near Infrared Coronagraphic Imager) on Gemini South and analysed using an in house, LOCI-like, post processing.
\end{abstract}

Keywords. planetary systems, instrumentation: adaptive optics, high angular resolution, techniques: image processing, stars: imaging, low-mass, brown dwarfs

\section{Introduction}

The Anglo-Australian Planet Search (AAPS) has been one of the major Doppler search programs, operating on the AAT since 1998. It currently targets some 250 stars for regular observation and has detected over 40 exoplanets to date. Some 13\% of our AAPS targets show evidence for long-period companions at periods greater than the baseline of our data. With their orbital periods only constrained to be longer than the AAPS survey time, we are unable to use the observed velocities over that period to constrain the masses of these objects: they could have periods of $\sim 20$ years and orbital separations of $\sim 6 \mathrm{AU}$, and hence planetary masses, or they could have periods of hundreds of years, separations of $>10 \mathrm{AU}$ and hence brown-dwarf or M-dwarf masses.

Direct imaging is the only means by which we can determine the nature of the longperiod candidates in our AAPS sample. We select all AAPS stars within 60pc, giving us 30 targets. Most of the AAPS targets are 4-6 Gy old G dwarfs, inactive and slowly rotating, providing good radial velocity stability. Having such an old target sample means we are unlikely to be able to detect planetary mass bodies, however, a NICI non-detection provides an upper mass limit, pushing targets towards the planetary mass regime. 
The detection of brown dwarf companions to these stars is also of great interest. The AAPS targets are mostly G dwarfs, for which metallicity, gravity and age can be better determined than any other class of star. Therefore, any brown dwarf companions discovered would break the age-mass degeneracy typically faced with un-agable field brown dwarfs and spectra/photometry could be used as benchmarks in this field.

\section{Observing Strategy and Data Reduction}

To perform our direct imaging search we use the Near Infrared Coronagraphic Imager (NICI) on Gemini South. In order to reach the the highest possible contrast we perform Angular Differential Imaging (ADI) (Marois et al. (2006)). To do this the telescopes de-rotator is switched off allowing the field to rotate on the detector but keeping the speckle noise stationary. In this way, the speckle noise can be fitted and removed from the observed data.

Our data reduction is described briefly below, for more details see Salter et al. in prep.

- Normal calibrations such as flat fielding, dark subtraction, bad pixel removal and de-warping are performed.

- NICI images show significant large scale structure which is fit and removed from each image.

- We perform our own implementation of a Locally Optimised Combination of Images (LOCI) analysis based largely the work of Lafreniere et al. (2007).

- Each image is de-rotated and combined to give the final result.

Short exposure images of some targets, prior to the main ADI observations, showed bright companions that have now been confirmed to be co-moving from further short integrations. Long ADI observations were not required for these targets. Three of our targets were not suitable for ADI observations as their location on the sky wouldn't give enough field rotation over a reasonable time frame. For these targets we used regular dithered imaging.

\section{Results}

To date, in our sample of 30, 11 targets have been found to have co-moving companions. All appear to be $\mathrm{M}$ dwarf companions at projected separations varying from $\sim 10-500 \mathrm{AU}$ (Salter et al. in prep). Twelve targets have been shown to not have any companions observable with NICI. Through analysis of the achieved contrast and inserting fake objects into the data, these observations provide an upper mass limit to the system. A lower mass limit and a minimum orbital period is also provided by the radial velocity measurements (Salter et al. in prep).

A further 7 targets have objects that have been detected but second epoch imaging is still required to confirm that they are co-moving.

\section{References}

Marois, C., Lafreniere, D., Doyon, R., Macintosh, B., \& Nadeau, D. 2006, ApJ, vol 641, 556-564 Lafreniere, D., Marois, C., Doyon, R., Nadeau, D., \& Artigau, E. 2006, ApJ, vol 660, 770-780 